\title{
Malignant Pleural Mesothelioma: An Update on the Role of Surgery
}

\author{
Maria Kalliopi Konstantinidou ${ }^{1}$, Christos Kakos ${ }^{1, ~ *, ~ D i m i t r i o s ~ K y p a r i s s o p o u l o s ~}{ }^{2}$ \\ ${ }^{1}$ Department of Cardiothoracic Surgery, Harefield Hospital, London, UK \\ ${ }^{2}$ Department of Thoracic Surgery, Mediterranean Hospital of Cyprus, Limassol, Cyprus
}

Email address:

m_klou@yahoo.gr (M. K. Konstantinidou), kakoschristos91@gmail.com (C. Kakos), dimkypar@hotmail.com (D. Kyparissopoulos)

*Corresponding author

\section{To cite this article:}

Maria Kalliopi Konstantinidou, Christos Kakos, Dimitrios Kyparissopoulos. Malignant Pleural Mesothelioma: An Update on the Role of Surgery. International Journal of Cardiovascular and Thoracic Surgery. Vol. 3, No. 6, 2017, pp. 78-85. doi: 10.11648/j.ijcts.20170306.14

Received: October 29, 2017; Accepted: December 4, 2017; Published: December 26, 2017

\begin{abstract}
Mesothelioma is a rare type of cancer which can occur in various sites, such as the peritoneum, the pericardium and tunica vaginalis testis; but malignant pleural mesothelioma is the most common type. Malignant pleural mesothelioma commonly affects older males that have been exposed to asbestos 20-40 years ago. The disease is difficult to be treated and has an overall survival expectancy of about 1 year. Histological subtypes include epithelioid, sarcomatoid and biphasic or mixed. Diagnosing this type of cancer is rather challenging and as a result it is usually diagnosed in most patients in progressed stages. Surgery with minimal procedures is applied to reach the diagnosis, with the Video Assisted Thoracoscopic procedure being considered to be the "gold standard". Patients with malignant pleural mesothelioma should be managed by experienced multidisciplinary teams, as treatment options include surgery, radiation therapy and /or chemotherapy. Extrapleural pneumonectomy and pleurectomy/decortication are the preferred procedures in the treatment of this malignancy, but whether they prolong life expectancy or improve quality of life of the patients still remains a controversial issue. In any case, careful assessment before surgery is of paramount importance. Multimodality approaches, which include surgery for patients who are fit, are often chosen in order to increase survival rates in clinical trials.
\end{abstract}

Keywords: Malignant Pleural Mesothelioma, Pleurectomy, Extra-Pleural Pneumonectomy, Surgery, Review

\section{Introduction}

Malignant Pleural Mesothelioma (MPM) is a rare entity, whose incidence has been increased over the last years. More than 2,500 Americans are diagnosed with MPM annually, while the incidence of MPM in males in Great Britain is 3.4/100.000. According to the World Health Organisation 125 million people have occupational exposure to asbestos. Mesothelioma can occur in different sites such as in the peritoneum, the pericardium or tunica vaginalis testis, but malignant pleural mesothelioma (MPM) is the most common type. $[1,2]$

Asbestos is considered to be the principal aetiological agent of MPM. Asbestos, a hydrated magnesium silicate fibrous mineral, refers to a group of six silicate minerals which are able to form very thin fibres: chrysotile, crocidolite, amosite, anthophyllite, tremolite and actinolite. However, the first studies which revealed association between asbestos and MPM were published in the 1960s, when Wagner et. al first described the disease in South Africans asbestos miners. [3]

\section{Epidemiology}

The incidence rates of MPM vary among different countries worldwide, as result mainly of the differences of asbestos import and consumption, but also of the different diagnostic approaches and awareness. The incidences vary from 7 per million in Japan to 40 per million in Australia inhabitants per year. [4] In USA the incidence is 10 per million people, a value which is declining since reaching a peak in the start of the century, while in Europe the incidence is approximately 20 per million, which is expected to achieve a maximum incidence before 2030. [5]

The proportion of MPM related to exposure to asbestos is $>80 \%$ in males but much less in females. Asbestos and 
MPM appear to have a dose-response relationship, but MPM may be observed in subjects having low-dose cumulative exposures. [6] MPM is associated with a history of occupational exposure to asbestos in $40-80 \%$ of patients. The incidence of MPM is $10 \%$ in asbestos workers. On the contrary, the incidence of MPM in the general population is estimated to be only $0.01-0.24 \%$. It is observed more frequently in men than women with a ratio $4: 1$, and has a peak incidence in the sixth and seventh decades of life. [7] While the vast majority of mesotheliomas are attributable to asbestos, a few other causes have been identified. Mesothelioma is a well-known complication of therapeutic radiation of lymphoma, breast cancer, lung cancer and other malignancies. [8] A genetic predisposition to mesothelioma in individuals exposed to the mineral erionite has been observed in the Turkish region of Cappadocia. [9] Inhabitants of North West villages of Greece in the area of Metsovo suffered from an increased incidence of malignant pleural mesothelioma, as they have been exposed since childhood to inhalation of asbestos, from a material containing tremolite, called "luto soil" that was used for white washing. [10] The role of Simian Virus 40 (SV40) in the pathogenesis of mesothelioma is controversial. [11, 12]

\section{Diagnosis}

Dyspnoea and chest pain are the first symptoms of a patient with MPM. Careful past medical and occupational history, revealing asbestos exposure is necessary for possible diagnosis of mesothelioma. Chest radiographs usually reveal unilateral pleural abnormalities with pleural effusion, while chest CT scan often demonstrates encasement of the lung by a thickened pleural peel. However, the diagnosis of MPM is tedious, as pleural plaques, while indicative for asbestos exposure, are not diagnostic. Moreover a diagnosis is achieved only in $26 \%$ of cases by repeated samples of pleural fluid cytology, as they are often negative. [13]

Video Assisted Thoracoscopic Surgery (VATS) has a high sensitivity up to $98 \%$ in diagnosing MPM according to existing literature, whereas Computed Tomography-guided needle biopsy has sensitivity of up to $88 \%$ and blind pleural biopsy's varies from $21 \%$ to $71 \%$. This information leads to the conclusion that the "Gold Standard" procedure to reach diagnosis is VATS. [14] In case thoracoscopic procedures are contraindicated or not available, ultrasound-guided true-cut biopsies can lead to diagnosis of MPM. [1]

According to the Recommendations of ERS/ESTS task force, thoracoscopy should be preferred for diagnostic investigation, as it allows complete visual examination of the pleura, multiple, deep and large biopsies and provides a diagnosis in $90 \%$ of cases (grade 1A).

\section{Histology}

Malignant pleural mesotheliomas arise from multipotential mesothelial or subserosal cells and exhibit a wide array of histologic patterns. There are three histologic subtypes of mesothelioma: epithelial, sarcomatoid and biphasic. The histologic appearance of MPM is easily confused with that of other neoplasms, and particularly the epithelial mesothelioma is difficult to distinguish from adenocarcinoma, and sarcomatoid mesothelioma from sarcoma. The epithelial subtype is the most common and carries the most favourable prognosis. [15] Epithelial mesotheliomas typically stain positive for calretinin, WT1, thrombomodulin, mesothelin and D2-40 and negative for carcinoembryonic antigen and thyroid transcription factor-1. Sarcomatoid mesothelioma can be differentiated from epithelioid mesothelioma and other sarcomatoid tumours as the immunohistochemistry study is positive for cytokeratin AE1/AE3 and CAM5.2 and negative for MyoD1, myoglobin desmin, a-SMA, S-100p etc. [16]

A recent systematic review and metanalysis proved the potential of circulating MicroRNAs as biomarkers in the diagnosis of MPM, which is a rather optimistic perspective in terms of early diagnosis and better prognosis in people exposed to asbestos or patients with MPM. [17]

\section{Prognosis - Staging}

Mesothelioma is a heterogeneous malignancy with a variety of other prognostic factors. The more widely used prognostic scoring system is this from the European Organization for Research and Treatment of Cancer. It identifies poor performance status, probable diagnosis of mesothelioma, serum leukocytosis, male gender, and sarcomatous subtype as poor prognostic indicators. The 1year survival rate in the patients with a good prognosis was $40 \%$, compared with $12 \%$ in those patients predicted to have a poor prognosis. [18]

A study of a Swiss centre for the treatment of MPM with induction chemotherapy and extrapleural pneumonectomy proposed a Multimodality Prognostic Score (MMPS) to identify whether patients would benefit from receiving multimodality treatment or not. The MMPS includes four variables: tumour volume, C-reactive protein levels, nonepithelioid histology and progressive disease. However, further study of these prognostic factors as well as other variables is necessary so that this prognostic score can be approved as a predictive test for patient selection. [19]

Patients with MPM should be managed by an experienced in MPM multidisciplinary team according to the NCCN guidelines. The treatment options for patients with MPM include surgery, radiation therapy (RT) and/or chemotherapy, while selected patients could be candidates for multimodality therapy.

Pre-treatment evaluation for patients diagnosed with MPM is done to stage patients and to assess whether patients are candidates for surgery. The evaluation process includes chest and abdominal CT with contrast and FDG-positron emission tomography (PET)-CT. If contralateral disease is suspected, then VATS can be considered. When possible, PET/CT scans should be obtained before pleurodesis, as talc causes pleural inflammation, which can affect FDG avidity. [20]

Mediastinoscopy and endobronchial ultrasonography 
(EBUS) fine needle aspiration (FNA) of the mediastinal lymph nodes is recommended, if surgical resection is being considered. [21] If suggested by imaging, laparoscopy may be done to exclude transdiaphragmatic extension (e.g. extension to peritoneum indicative of stage IV - unresectable disease) or chest magnetic resonance imaging (MRI).

For staging the International Mesothelioma Interest Group (IMIG) TNM staging system is approved by the American Joint Committee on Cancer (AJCC) [22]

Most patients present with advanced disease, although under-staging is common with PET-CT and makes it even more difficult to accurately stage patients before surgery. However PET-CT can be used to determine whether metastatic disease is present. [23]

Further evaluation for patients with clinical stage I-III MPM considered for surgery is performed by pulmonary function tests (PFTs), perfusion scanning (if FEV1 <80\%) and cardiac stress tests. Only patients with clinical stage I-III MPM, who are medically operable and can tolerate surgery, are recommended to undergo surgical resection. Trimodality therapy (i.e. chemotherapy, surgery and RT) is recommended for patients with clinical stage I-III MPM who are medically operable. Chemotherapy alone is recommended for those who are not operable, those with clinical stage IV MPM, or those with sarcomatoid histology according to the NCCN Guidelines for MPM.

\section{Surgical Procedures}

Surgery for MPM includes not only procedures for diagnosis and staging, but also debulking operations that aim palliation or extensive cytoreductive procedures. The reduction of intrathoracic tumour burden to microscopic levels is expected to increase survival and improve quality of life. While minor procedures evolve mediastinoscopy and VATS, cytoreductive procedures are achieved either by extrapleural pneumonectomy (EPP) or pleurectomy / decortication in various extents.

Pleural effusions could be managed using thoracoscopic talc pleurodesis or placement of a drainage catheter. Thoracentesis can also be used to remove pleural fluid in order to decrease dyspnoea either prior to treatment or for those patients who are not candidates for more aggressive treatment. [24]

As mesothelioma surgeons might interpret the aforementioned procedures in different ways, as to their extent - resection of the entire parietal and visceral pleura, with or without partial resection of the pericardium or diaphragm if involved by tumour- the use of a well-defined terminology appears to be necessary.

On this context, the IASLC Mesothelioma Domain and the International Mesothelioma Interest Group (IMIG), based on a survey data, which represented the opinions of experienced MPM surgeons from multiple centres in different geographical regions, have recommended the following terminology [25] to be used in the forthcoming Mesothelioma Staging Project: a. Extrapleural pneumonectomy (EPP): en bloc resection of the parietal and visceral pleura with the ipsilateral lung, pericardium, and diaphragm. In cases where the pericardium and/or diaphragm are not involved by tumour, these structures may be left intact.

b. Extended Pleurectomy/Decortication (e-P/D): parietal and visceral pleurectomy to remove all gross tumours with resection of the diaphragm and/or pericardium. The IASLC Mesothelioma Domain suggests use of the term "extended" rather than "radical" in this instance as the latter implies a completeness of resection with added therapeutic benefit. There is currently insufficient evidence that resection of the pericardium and diaphragm provides either.

c. Pleurectomy/Decortication (P/D): parietal and visceral pleurectomy to remove all gross tumours without diaphragm or pericardial resection.

d. Partial pleurectomy (PP): partial removal of parietal and/or visceral pleura for diagnostic or palliative purposes but leaving gross tumour behind.

It is essential that patients receive a careful assessment before surgery is performed. Mediastinal lymph node dissection is recommended in patients having either $\mathrm{P} / \mathrm{D}$ or EPP. In patients who are medically operable, the decision whether to perform a P/D or an EPP may not be made until surgical exploration.

The optimal surgical procedures for mesothelioma, as well as the appropriate role of surgical resection in this disease remain quite controversial.

According to the ERS/ESTS TASK FORCE, debulking pleurectomy/decortication can be defined as significant but incomplete macroscopic clearance of pleural tumour. The objective of the operation is to relieve an entrapped lung by removing the visceral tumour cortex. Removal of the parietal tumour cortex may relieve a restrictive ventilatory deficit and reduce chest wall pain. The operative procedure may be performed by either open thoracotomy or VATS.

The evidence supporting debulking surgery over chemical pleurodesis is controversial. There are small series of retrospective studies which provide low-grade evidence for debulking pleurectomy. [26-29] It is accepted that the associated morbidity of thoracotomy may diminish the benefits; [30] however there is limited but emerging evidence that VATS can not only provide good symptom control but may also have a beneficial effect on survival. [28]

In 2014 the results of an open-label, randomised, controlled trial were announced. Rintoul et al performed a phase III clinical trial, which was supported by the National Cancer Research Institute, comparing the efficacy of VATS partial pleurectomy (VATS-PP) versus talc pleurodesis in 196 patients with MPM and pleural effusion. The overall survival at 1 year was $52 \%$ in VATS-PP group comparing to the talc pleurodesis group in which it was $57 \%$. Regarding the complications of each group, the VATS-PP group had almost double the amount of complications accounting for $31 \%$ of the patients in contrast to only $14 \%$ of the talc pleurodesis patients. Moreover the length of hospital stay was significantly longer in the first group (7 
days) than on the second (3 days). The MesoVATS trial concluded that VATS-PP does not improve overall survival in patients with pleural effusion secondary to MPM, although it allows more adequate control of pleural effusion and better quality of life in 6 and 12 months. VATS talc pleurodesis is superior in terms of overall survival, complication and hospital stay. [31]

Pleurectomy/decortication can be considered in patients to obtain symptom control, especially in symptomatic patients with entrapped lung syndrome who cannot benefit from chemical pleurodesis but should not be proposed in a curative intent (grade 2C). The VATS approach is preferred (grade 1C).

Cao et al performed a systematic review to estimate and compare the efficacy of different pleurectomy and decortication techniques, including extended $\mathrm{P} / \mathrm{D}, \mathrm{P} / \mathrm{D}$ and PP. 1916 patients from 34 different studies were included in the results. The interpretation of the results showed a longer overall and disease-free survival in the e-P/D group, although the perioperative morbidity and length of stay was higher in this group compared to P/D and PP. [32]

Extended (cytoreductive) surgery is defined as an attempt to remove all macroscopic disease from the hemithorax with a view to cure the patient with MPM and includes EPP and extended $\mathrm{P} / \mathrm{D}$. The preference and the benefit of these two techniques is a matter surrounded by rather great controversy

Another systematic review and meta-analysis of the existing literature conducted by Cao et al in 2014 compared the perioperative mortality and morbidity between patients who underwent either EPP or e-P/D. Perioperative mortality $(2.9 \%$ vs $6.8 \%, \mathrm{p}=0.02)$ and morbidity $(27.9 \%$ vs $62.0 \%$, $\mathrm{p}<0.0001)$ were significantly lower in e-P/D compared to EPP. The median overall survival was 12-22 months for EPP, whereas it was slightly better (13-29 months) for the e-P/D. It is noteworthy that the extended surgical treatment was part of multimodality therapy in all patients. [33]

The efficacy of extensive surgery for mesothelioma is controversial due to limited evidence. Among resected mesothelioma patients, the only published long-term survivors have undergone radical surgery (EPP) as part of a multimodality programme.

Regarding the efficiency of EPP a recent multicentre retrospective study of 518 patients who underwent EPP as part of a multimodality treatment for MPM demonstrated an overall survival of 18 months, with perioperative morbidity $26.3 \%$ and mortality within 90 days of $6.9 \%$. This particular study also concluded that female patients, epithelioid histology and induction chemotherapy favour the overall survival after EPP. [34]

A number of subsequent prospective and retrospective series that are reported have all demonstrated a similar median survival of 20-24 months. Operative mortality has fallen to an acceptable level of 5\% in experienced centres but morbidity remains as high as $50 \%$. [35, 36, 37]

According to another review on the treatment of MPM by A. Wolf and R. Flores, the selection of the cytoreductive procedure is influenced by a variety of patient-specific and surgeon-specific or centre-specific factors. Also perioperative mortality and morbidity have a lower percentage of occurrence in favour of P/D compared to EPP. [38]

A recent meta-analysis tried to give an answer on the prominent question regarding which technique (EPP or P/D) should be the first choice in the surgical treatment of MPM. After analysing data of 1512 patients who were treated with P/D and 1391 patients who were treated with EPP, they deduced that $\mathrm{P} / \mathrm{D}$ offers lower short-term mortality (30-day) than EPP $(4.5 \%$ vs $1.7 \%, \mathrm{p}<0.05)$. However the 2 -year survival, although superior in the $\mathrm{P} / \mathrm{D}$ group, was not statistically significant. Despite a variety of limitations this study suggested performing P/D over EPP in MPM patients. [39]

The multicenter MARS-2 trial, which is currently in the recruiting process, aims to prove whether $\mathrm{P} / \mathrm{D}$ improves overall survival over no surgery. [40]

According to the ERS/ESTS Task Force recommendations, radical surgery (EPP) should only be performed in clinical trials, in specialised centres, as part of multimodality treatment.

The ESMO Guidelines Committee suggested that EPP or $\mathrm{P} / \mathrm{D}$ should be used to perform macroscopic resection (R1 resection) of the tumour. In addition, cytoreductive surgery should be part of a multimodality treatment in specialized centres as part of a study. [1]

As indicated by literature from previous years, surgery alone for MPM is not curative since no oncological resection margins can be obtained. The pleural lining, especially on the pericardium and mediastinum, cannot be resected easily with a 1-2-cm margin. Therefore, all surgical procedures are considered R1 resections. [36] The abovementioned conclusion, based on observation, has led to the implementation of the rationale for combined therapy - with strong but low quality level of evidence.

Multimodality approach for MPM consists of three components: cytoreductive surgery (EPP or P/D) to achieve macroscopic complete resection, adjuvant/neo-adjuvant chemotherapy and adjuvant/neo-adjuvant radiotherapy. [41]

A recent analysis of 663 consecutive patients treated with EPP or P/D highlighted the previous observations. There was no statistical difference in survival by procedure at any stage. The 5-year overall survival rate was only $12 \%$. Patients who underwent $\mathrm{P} / \mathrm{D}$ had a higher local recurrence rate $(65 \%)$, while distant recurrences $(66 \%)$ predominated in patients who underwent EPP. The operative mortality rate was slightly lower for P/D vs EPP (4\% vs 7\%, respectively). By univariate analysis, statistically superior survival was associated with stage, epithelioid histology, P/D, multimodality therapy, and female gender. On multivariate analysis, the survival benefit observed for $\mathrm{P} / \mathrm{D}$ was only marginally significant (hazard ratio [HR], 1.4). [42]

A multicentre study reported a median survival time of 23 months for the $74 \%$ of patients who successfully underwent EPP after receiving neo-adjuvant chemotherapy. [43]

Krug et al performed a trial, published in 2009, which included 77 patients who received chemotherapy, $70 \%$ of them underwent an EPP and 40 of those received radiation. 
The median overall survival was only 16.8 months. However the median survival in the group of patients who completed the trimodality therapy was 29.1 months. A conclusion was reached that the trimodality approach benefits the survival of patients with MPM. [44]

A Swiss centre analysed the data of 186 patients with MPM treated over 12 consecutive years with induction chemotherapy followed by EPP. Only $52 \%$ of the patients received adjuvant radiotherapy. Induction chemotherapy was either cisplatin/gemcitabine or cisplatin/pemetrexed, with the second group having significantly fewer haematological complications. 128 patients underwent EPP with a median overall survival of 22 months and 30-days mortality of $4.7 \%$. In comparison, patients treated without EPP had a worse median overall survival of 11 months. This case series showed a clear and significant superiority of multimodality therapy with induction chemotherapy, EPP, plus/minus adjuvant radiotherapy in patients with MPM. [19]

The Mesothelioma and Radical Surgery (MARS) trial, published in 2011, tried to evaluate the clinical outcomes of patients who were randomized to have EPP (24 patients) or no EPP (26 patients) after induction platinum-based chemotherapy. The analysis of the results led to the conclusion that EPP as part of trimodality therapy over chemotherapy alone had no better outcomes in terms of 12months survival (EPP $52.2 \%$ vs no EPP 73.1\%) and demonstrated a perioperative mortality of $18 \%$. [45]

However, since being published, this trial received a lot of criticism as the number of the patients recruited was relatively small to extract safe conclusions regarding the superiority of the EPP or no-EPP approach. Additionally, patients did not receive a standardised chemotherapy scheme on a specific dose and timing before the operation. The $18 \%$ of perioperative mortality in the group of patients who underwent EPP as part of the MARS trial is significantly higher than the mortality reported by a variety of other recent trials accounting for $0-5 \%$. [46]

A retrospective study in 540 patients reported that several factors, such as EPP, surgeon experience and pemetrexed increased survival for select patients. [47] The NCCN panel and other clinicians recommended EPP for selected low-risk patients but not for patients with comorbidities. [48]

A systematic review of the efficacy of trimodality therapy with neoadjuvant chemotherapy, EPP and adjuvant radiotherapy showed a potential benefit from this approach in highly experienced institutions. It was also concluded that the use of EPP to treat patients with MPM has an acceptable perioperative mortality rate in these institutions and, thus, should be utilized. [49]

The integration of chemotherapy into a multimodality treatment strategy has been evaluated in the past, during and after surgery, but current trials usually adopt a neoadjuvant approach, including a platinum agent with pemetrexed, followed by surgery. Some but not all trials mandate radiation therapy after resection. Recent data have suggested that the use of radiation $21 \mathrm{~Gy}$ in three fractions - used to decrease the potential risk of seeding from thoracoscopy sites
- is ineffective and unnecessary. [50]

The same conclusion was reached by another randomised, international, multicentre phase 2 trial which compared the outcome of neoadjuvant chemotherapy and extrapleural pneumonectomy with or without hemithoracic radiotherapy. [51]

According to SMART Trial, the aim of which was to evaluate the necessity and benefit of prophylactic radiotherapy after surgical and large-bore pleural procedures, routine use of prophylactic radiotherapy is not justified. [52]

A recent article commented on the factors influencing the selection of the appropriate treatment in patients with MPM and suggested that prolonged survival will only be achieved, if all parts of the cutoreductive-based multimodality treatment protocol would be undertaken. Furthermore, it concluded that achieving macroscopic complete resection, while simultaneously reducing the morbidity and mortality of the cytoreductive surgical approaches is a cornerstone in the pursuit of a longer disease-free overall survival in patients with MPM. Finally, a proposal was made, to utilize intraoperative techniques (photodynamic therapy or hyperthermic pleural lavage with povidone-iodine or hyperthermic intraoperative chemotherapy) as an inextricable part of the multimodality treatment to improve regional control of the disease. [41]

\section{Conclusions}

According to the NCCN Guidelines Version 2014 for Malignant Pleural Mesothelioma

Surgical resection should be performed on carefully evaluated patients by board certified thoracic surgeons. For patients being considered for surgery, a single port thoracoscopy on the line of the potential incision is recommended.

The aim of surgery is complete gross cytoreduction of the tumour. When this is not possible, such as multiple sites of chest wall invasion, surgery should be aborted. The surgical choices are either pleurectomy/decortication (P/D) with mediastinal lumph node sampling or extrapleural pneumonectomy (EPP). Mediastinal node sampling should be performed.

EPP may be the best option for early stage (confined to the pleural envelope, no N2 lymph node involvement) with favourable history (epithelioid) in low- risk patients. For advanced disease, (high nodal disease, areas of local invasion) mixed histology and/or high risk patients, pleurectomy/decortication may be a better choice. After recovery from surgery, patients should be referred for adjuvant therapy which may include chemotheraphy and radiation therapy depending on whether any neoadjuvant therapy was used and on the pathological analysis of the surgical specimen.

According to ESMO Guidelines Committee surgery (Partial pleurectomy) is indicated for palliation of pleural effusion when chest tube drainage is not successful and for diagnosis and staging of the patient EPP or $\mathrm{P} / \mathrm{D}$ should be 
used as part of multimodality treatment and specifically as part of a study. The aim of either EPP or P/D should be to perform macroscopically complete resection.

Despite recent advances in the treatment of mesothelioma which are attributed to increased studies that are reported lately, malignant pleural mesothelioma still has poor prognosis. For selected patients that are eligible for resection and multimodality therapy, survival has improved. The advance in surgical techniques, but also the use of uniform definition of surgical techniques could lead to multicentre studies with the aim to prolong expectancy and quality of life of patients with malignant pleural mesothelioma. Development of biomarkers and molecular targeted agents could yield new treatment options and early detection of mesothelioma allowing complete resection which in combination with multimodality therapy, could make long term eradication of the disease and cure feasible.

\section{References}

[1] P. Baas, D. Fennell, K. M. Kerr, P. E. Van Schil, R. L. Haas \& S. Peters, on behalf of the ESMO Guidelines Committee. Malignant pleural mesothelioma: ESMO Clinical Practice Guidelines for diagnosis, treatment and follow-up. Ann Oncol. 2015 Sep; 26 Suppl 5:v31-9. doi: 10.1093/annonc/mdv199. Epub 2015 Jul 28.

[2] Asbestos: elimination of asbestos-related diseases. Fact sheet N³43 World Health Organisation, 2016. Available online: http://www.who.int/mediacentre/factsheets/fs343/en/index.ht $\mathrm{ml}$.

[3] Wagner JC, Sleggs CA, Marchand P. Diffuse pleural mesothelioma and asbestos exposure in the North Western Cape Province. Br J Ind Med 1960; 17: 260-271.

[4] Robinson BW, Lake RA. Advances in malignant mesothelioma. N Engl J Med 2005; 353: 1591-1603. doi:10.1056/NEJMra050152.

[5] Jing Ai and James P. Stevenson Current Issues in Malignant Pleural Mesothelioma Evaluation and Management Oncologist. 2014 Sep; 19 (9): 975-984. doi: 10.1634/theoncologist.2014-0122.

[6] A. Scherpereel, P. Astoul, P. Baas, T. Berghmans, H. Clayson, P. de Vuyst, et al. ERS/ESTS TASK FORCE. Guidelines of the European Respiratory Society and the European Society of Thoracic Surgeons for the management of malignant pleural mesothelioma. Eur Respir. J. 2010; 35: 479-495 doi: 10.1183/09031936.00063109.

[7] M. T. Truong, C. Viswanathan, MB. C. Godoy, Brett W. Carter, EM. Marom. Malignant Pleural Mesothelioma: Role of CT, MRI, and PET/CT in Staging Evaluation and Treatment Considerations doi: 10.1053/j.ro.2013.03.017.

[8] Hodgson DC, Gilbert ES, Dores GM, Schonfeld SJ, Lynch CF Storm $\mathrm{H}$, et al, Long-term solid cancer risk among 5-year survivors of Hodgkin's lymphoma. J Clin Oncol 2007; 25: 1489-1497.

[9] Roushdy-Hammady I, Siegel J, Emri S, et al. Genetic susceptibility factor and malignant mesothelioma in the Cappadocian region of Turkey. Lancet 2001; 357: 444-445 doi: http://dx.doi.org/10.1016/S0140-6736 (00)04922-9.

[10] K. Sakellariou, V. Malamou-Mitsi, A. Haritou, C. Koumpaniou, C. Stachouli, I. D. Dimoliatis, S. H. Constantopoulos. Malignant pleural mesothelioma from nonoccupational asbestos exposure in Metsovo (north-west Greece): slow end of an epidemic? Eur Respir J., 1996, 9, 1206-1210.

[11] Cristaudo A, Foddis R, Vivaldi A, et al. SV40 enhances the risk of malignant mesothelioma among people exposed to asbestos: a molecular epidemiologic case-control study. Cancer Res 2005; 65: 3049-3052.

[12] Manfredi JJ, Dong J, Liu WJ, et al. Evidence against a role for SV40 in human mesothelioma. Cancer Res 2005; 65: 26022609.

[13] M. Ray, H. L. Kindler. Malignant pleural mesothelioma. An Update on Biomarkers and Treatment. Chest 2009; 136: 888896 doi: 10.1378/chest.08-2665.

[14] Shivani C Patel and Jonathan E Dowell Modern management of malignant pleural mesothelioma. Lung Cancer (Auckl). 2016; 7: 63-72. doi: 10.2147/LCTT. S83338.

[15] Ordonez NG Immunohistochemical Diagnosis of epithelioid mesothelioma: an update. Arch Pathol Lab Med 2005; 129: 1407-1414.

[16] Kouki Inai Pathology of mesothelioma Environ Health Prev Med. 2008 Mar; 13 (2): 60-64. doi: 10.1007/s12199-007-0017-6.

[17] Luigina Micolucci, Most Mauluda Akhtar, Fabiola Olivieri, Maria Rita Rippo, and Antonio Domenico Procopio Diagnostic value of microRNAs in asbestos exposure and malignant mesothelioma: systematic review and qualitative meta-analysis Oncotarget. 2016 Sep 6; 7 (36): 58606-58637. doi: 10.18632 /oncotarget.9686.

[18] Curran D, Sahmoud T, Therasse P, et al. Prognostic factors in patients with pleural mesothelioma: the European Organization for Research and Treatment of Cancer experience. J Clin Oncol 1998; 16: 145-152.

[19] Opitz I, Friess M, Kestenholz P, Schneiter D, Frauenfelder T, Nguyen-Kim TD, et al. A New Prognostic Score Supporting Treatment Allocation for Multimodality Therapy for Malignant Pleural Mesothelioma: A Review of 12 Years' Experience. J Thorac Oncol. 2015 Nov; 10 (11): 1634-41 doi: 10.1097/JTO.0000000000000661.

[20] Pilling J, Dartnell JA, Lang- Lazdunski L. Integrated positron emission tomography-computed tomography does not accurately stage intrathoracic diseaseof patients undergoing trimodality therapy for malignant pleural mesothelioma. Thorac Cardiovas Surg 2010; 58: 215-219.

[21] Rice DC, Steliga MA, Stewart J, et al. Endoscopic ultrasound guided fine needle aspiration for staging of malignant pleural mesothelioma. Ann Thorac Surg 2009; 88: 862-869. doi: 10.1016/j.athoracsur.2009.05.022.

[22] Edge SB, Byrd DR, Compton CC, et al. AJCC Cancer Staging Manual, $7^{\text {th }}$ edition. New York: Springer; 2010.

[23] Wilcox BE, Supramaniam RM, Paller PJ, et al. Utility of integrated computed tomography- positron emission tomography for selection of operable malignant pleural mesothelioma. Clin Lung Cancer 2009; 10: 244-248 doi: 10.3816/CLC.2009.n.033. 
[24] Aelony Y, Yao JF. Prolonged survival after talk poudrage for malignant pleural mesothelioma: case series. Respirology 2005; 10: 649-655.

[25] Rice D, Rusch V, Pass H, Asamura H, Nakano T, Edwards J, et al. Recommendations for Uniform Definitions of Surgical Techniques for Malignant Pleural Mesothelioma. A Consensus Report of the International Association for the Study of Lung Cancer International Staging Committee and the International Mesothelioma Interest Group Journal of Thoracic Oncology, VOL 6, Number 8, August 2011 doi: 10.1097/JTO.0b013e3182208e3f.

[26] Waller DA, Morritt GN, Forty J. Video-assisted thoracoscopic pleurectomy in the management of malignant pleural effusion. Chest 1995; 107: 1454-1456.

[27] Soysal O, Karaoglanoglu N, Demiracan S, et al. Pleurectomy/ decortication for palliation in malignant pleural mesothelioma: results of surgery. Eur J Cardiothorac Surg 1997; 11: 210-213.

[28] J C Halstead 1, E Lim, R M Venkateswaran, S C Charman, M Goddard, A J Ritchie. Improved survival with VATS pleurectomy-decortication in advanced malignant mesothelioma. Eur J Surg Oncol 2005; 31: 314-320. doi: 10.1016/j.ejso.2004.08.014.

[29] Apostolos Nakas 1, Antonio E Martin Ucar, John G Edwards, David A Waller. The role of video assisted thoracoscopic pleurectomy/decortication in the therapeutic management of malignant pleural mesothelioma. Eur J Cardiothorac Surg 2008; 33: 83-88. doi: 10.1016/j.ejcts.2007.09.039.

[30] Martin-Ucar AE, Edwards JG, Rengajaran A, et al. Palliative surgical debulking in malignant mesothelioma. Predictors of survival and symptom control. Eur J Cardiothorac Surg 2001; 20: $1117-1121$.

[31] Rintoul RC, Ritchie AJ, Edwards JG, Waller DA, Coonar AS, Bennett M, et al, MesoVATS Collaborators. Efficacy and cost of video-assisted thoracoscopic partial pleurectomy versus talc pleurodesis in patients with malignant pleural mesothelioma (MesoVATS): an open-label, randomised, controlled trial. Lancet. 2014 Sep 20; 384 (9948): 1118-27. doi: 10.1016/S0140-6736 (14)60418-9.

[32] Cao C, Tian DH, Pataky KA, Yan TD Systematic review of pleurectomy in the treatment of malignant pleural mesothelioma. Lung Cancer. 2013 Sep; 81 (3): 319-27 doi: 10.1016/j.lungcan.2013.04.024.

[33] Cao C, Tian D, Park J, Allan J, Pataky KA, Yan TD, A systematic review and meta-analysis of surgical treatments for malignant pleural mesothelioma. Lung Cancer. 2014 Feb; 83 (2): 240-5 doi: 10.1016/j.lungcan.2013.11.026

[34] Spaggiari L, Marulli G, Bovolato P, Alloisio M, Pagan V, Oliaro A, et al. Extrapleural Pneumonectomy for Malignant Mesothelioma: An Italian Multicenter Retrospective Study Ann Thorac Surg. 2014 Jun; 97 (6): 1859-65 doi: 10.1016/j.athoracsur.2014.01.050.

[35] Sugarbaker DJ, Jaklitsch MT, Bueno R, et al. Prevention, early detection, and management of complications after 328 consecutive extrapleural pneumonectomies. J Thorac Cardiovasc Surg 2004; 128: 138-146. doi: 10.1016/j.jtcvs.2004.02.021.

[36] Rusch VW, Rosenzweig K, Venkatraman E, et al. A phase II trial of surgical resection and adjuvant high-dose hemithoracic radiation for malignant pleural mesothelioma. J Thorac
Cardiovasc Surg 2001; 122: 788-795. doi: $10.1067 / \mathrm{mtc} .2001 .116560$.

[37] Weder W, Stahel RA, Bernhard J, et al. Multicenter trial of neoadjuvant chemotherapy followed by extrapleural pneumonectomy in malignant pleural mesothelioma. Ann Oncol 2007; 18: 1196-1202. doi: 10.1093/annonc/mdm093.

[38] Wolf AS, Flores RM. Current Treatment of Mesothelioma: Extrapleural Pneumonectomy Versus Pleurectomy/Decortication. Thorac Surg Clin. 2016 Aug; 26 (3): 359-75 doi: 10.1016/j.thorsurg.2016.04.003.

[39] Taioli E, Wolf AS, Flores RM Meta-analysis of survival after pleurectomy decortication versus extrapleural pneumonectomy in mesothelioma. Ann Thorac Surg. 2015 Feb; 99 (2): 472-80 doi: 10.1016/j.athoracsur.2014.09.056.

[40] MARS 2: A Feasibility Study Comparing (Extended) Pleurectomy Decortication Versus no Pleurectomy Decortication in Patients With Malignant Pleural Mesothelioma (MARS2) NCT02040272. https://clinicaltrials.gov/ct2/show/NCT02040272 Date last updated: May 11, 2017. Date last accessed: May 2017.

[41] Wald O, Sugarbaker DJ. Malignant pleural mesothelioma: key determinants in tailoring the right treatment for the right patient. J Thorac Dis. 2017; 9 (3): 485-489. doi:10.21037/jtd.2017.03.27.

[42] Flores RM, Pass H, Seshan VE, Dycoco J, Zakowski M, Carbone $\mathrm{M}$, et al. Extrapleural pneumonectomy versus pleurectomy/decortication in the surgical management of malignant pleural mesothelioma: results in 663 patients. J Thorac Cardiovasc Surg 2008; 135: 620-626 doi: 10.1016/j.jtcvs.2007.10.054.

[43] Weder W, Stahel RA, Bernhard J, et al. Multicenter trial of neo-adjuvant chemotherapy followed by extrapleural pneumonectomy in malignant pleural mesothelioma. Ann Oncol 2007; 7: 1196-1202 doi: 10.1093/annonc/mdm093.

[44] Krug LM, Pass HI, Rusch VW, Kindler HL, Sugarbaker DJ, Rosenzweig KE, et al. Multicenter phase II trial of neoadjuvant pemetrexed plus cisplatin followed by extrapleural pneumonectomy and radiation for malignant pleural mesothelioma. J Clin Oncol 2009; 27: 3007-3013 doi: 10.1200/JCO.2008.20.3943.

[45] Treasure T, Lang-Lazdunski L, Waller D, Bliss JM, Tan C, Entwisle J, et al; MARS trialists. Extra-pleural pneumonectomy versus no extra-pleural pneumonectomy for patients with malignant pleural mesothelioma: clinical outcomes of the Mesothelioma and Radical Surgery (MARS) randomised feasibility study. Lancet Oncol. 2011 Aug; 12 (8): 763-72. doi: 10.1016/S1470-2045 (11)70149-8.

[46] Weder W, Stahel RA, Baas P, Dafni U, de Perrot M, McCaughan BC. The MARS feasibility trial: conclusions not supported by data. Lancet Oncol. 2011 Nov; 12 (12): 1093-4. doi: 10.1016/S1470-2045 (11)70307-2.

[47] Yan TD, Cao CQ, Boyer $M$ et al. Improving survival results after surgical management of malignant pleural mesothelioma: an Australian institution experience. Ann Thorac Cardiovasc Surg 2011; 17: 243-249.

[48] Zauderer MG, Krug LM. The evolution of multimodality therapy for malignant pleural mesothelioma. Curr Treat Options Oncol 2011; 12: 163-172 doi: 10.1007/s11864-0110146-4. 
[49] Cao C, Tian D, Manganas C et al. Systematic review of trimodality therapy for patients with malignant pleural mesothelioma. Ann Cardiothorac Surg 2012; 1: 428 - 437. doi: 10.3978/j.issn.2225-319X.2012.11.07.

[50] Davies HE, Musk AW, Lee YC. Prophyllactic radiotherapy for pleural puncture sites: the controversy continues. Curr Opin Pulm Med 2008; 14: 326-330 doi: 10.1097/MCP.0b013e3282fcea50.

[51] Stahel RA, Riesterer O, Xyrafas A, Opitz I, Beyeler M, Ochsenbein A, et al. Neoadjuvant chemotherapy and extrapleural pneumonectomy of malignant pleural mesothelioma with or without hemithoracic radiotherapy (SAKK 17/04): a randomised, international, multicentre phase 2 trial. Lancet Oncol. 2015 Dec; 16 (16): 1651-8. doi: 10.1016/S1470-2045 (15)00208-9.
[52] Clive AO, Taylor H, Dobson L, Wilson P, de Winton E, Panakis N, Pepperell J, Howell T, Stewart SA, Penz E, Jordan $\mathrm{N}$, Morley AJ, Zahan-Evans N, Smith S, Batchelor TJ, Marchbank A, Bishop L, Ionescu AA, Bayne M, Cooper S, Kerry A, Jenkins P, Toy E, Vigneswaran V, Gildersleve J, Ahmed M, McDonald F, Button M, Lewanski C, Comins C, Dakshinamoorthy M, Lee YC, Rahman NM, Maskell NA Prophylactic radiotherapy for the prevention of proceduretract metastases after surgical and large-bore pleural procedures in malignant pleural mesothelioma (SMART): a multicentre, open-label, phase 3, randomised controlled trial. Lancet Oncol. 2016 Aug; 17 (8): 1094-104. doi: 10.1016/S1470-2045 (16)30095-X. 